\title{
STOPINCH UND MARKT
}

\section{HANSEKAUfLEUTE AUS BRECKERFELD UND UMGEBUNG}

\author{
von Klaus Albert Höller
}

\section{Eine Urkunde von 1408}

Im Archiv der Evangelischen Kirchengemeinde in Breckerfeld befindet sich unter der Signatur U1 einc Urkunde, welche am 3. November 1408 in London ausgestellt wurde. Ein Evert Stopinch übergibt sein gesamtes Erbe am Hof Stoupingh im Kirchspiel Breckerfeld an einen Johannes Markt. Diese Urkunde und ihr Inhalt sind der Hanseforschung bisher nicht bekannt gewesen. In dem prosopographischen Katalog der in der zweiten Hälfte des 14. Jahrhunderts in Brügge nachweisbaren Hansekaufleute finden sich zwar jeweils Familienangehörige der Beteiligten, aber keine Angaben zu dem Vorgang von 1408.'

600 Jahre nach diesem Vorgang soll die Urkunde hier im Wortlaut veröffentlicht und in die Rahmen der bisher bekannten Familiengeschichten gestellt werden. Darüber hinaus bietet sich die Gelegenheit, das Wirken der Hansekaufleute aus einer der kleinsten Hansestädte und ihrer ländlichen Umgebung darzustellen, das bisher weitgehend unbekannt war bzw. nicht im Zusammenhang gesehen werden konnte. Die Forschung z. B. in Kiel oder Greifswald vermag die vielen Familiennamen, die ja weitgehend auch Hofnamen sind, nur schwer räumlich einzuordnen. So konnte z. B. Asmussen 1989 zwar die Namen Breckerfeld, Remlingrade und Feckinghausen kartieren, die Kaufleute in der Halle/(Wüstung), Heetvelt/ Hedfeld, van der Hol/Hohle/Holle, Craywinkel/Krähwinkel, Hosenberghe/Osenberg, Scupinc/Steupingen und Walevelt/Vahlefeld (14. Jh./heute) jedoch nicht verorten. Andererseits gibt es unter den Breckerfeldern

\footnotetext{
' Werner Paravicini und Horst Wernicke (Hg.), Hansekaufleute in Brügge, Teil 3: Prosopographischer Katalog zu den Brügger Steuerlisten (1360-1390); bearb. von Ingo DIERCK, Sonja DÜNNEBEIL und Renée RÖSSNER (Kieler Werkstücke Reihe D Bd. 11), I Frankfurt/M. 1999. Nr. 229/229. I, 315/315; s. auch ebd. Hansekaufleute in Brügge 1358-1408, Teil 2: Georg ASMUSSEN, Die Lübecker Flandernfahrer in der zweiten Hälfte des 14. Jahrhunderts (1358-1408), Frankfurt/M. 1999, Karte 2, S. 90.
} 
keine Historiker und sie wohnen fern der großen Bibliotheken. Aber erst das Zusammenspiel der übergreifenden Hochschulforschung mit der lokalen Überlieferung ergibt das Gesamtbild.

Kundich sy allen luden dey disse breff seyn solen offte horen lesen dat ich

Evert Stopinch geve unde hebbe gegeven myn vaderlik unde moderlich Erve geheyten

Stoupingh in brekelvelde kespelle to Johannes Market al myn Recht dat my an ge

vallen is offe an vallen mach In tiden to te Comonde sinen willen mede to doene

to vorcopene unde to vorgevene also en dunket dat eme best es Dit hebbe ich

Evert gedaen mit vrien willen unde gesundeme lyve unbetwunge unde

ungevangen und ungebunden und sy dit bekannt vür alle luden sunder allen

kümmer und argelist und ich Evert unt Erve my [unleserlich] und min Erffna

men van allet dat ich dar ane hebben offt hebben mochte nu off hir na und

Johannes market vorgenannt es dar mede geErvet und syne Erfnamen nu und

to Ewigen tiden unde dagen na comende und al dat Recht dat ich dar van gehat solden hebben gehat und noch hebben mochte so vorgescreven stet In Tuch

nisse der warheyt so hebbich Evert vorgescreven myn seghel op spatium breyffs gedrucht datum London anno domini xiiii ${ }^{C}$ viii ${ }^{\circ} i^{\circ}$ die novembris

Die Urkunde Ul ist die älteste im Kirchenarchiv, ohne weitere Zusätze, etwa wie sie hierhin kam und von wem und wann. Das Pergament blieb leidlich erhalten, ebenso die gut leserliche Schrift, nicht zuletzt wegen deren Größe, das Siegel ist allerdings so zerstört, dass sein Inhalt nicht zu deuten ist. Das Pergament hat die Größe von $26,5 \times 19 \mathrm{~cm}$, der Satzspiegel, der 14 Zeilen beträgt, $22,5 \times 13 \mathrm{~cm}$, darunter auf das Pergament aufgedrückt das Siegel. Die mittelniederdeutsche Sprache des Hanseraumes bereitet der Deutung keine Schwierigkeiten. Der Schreiber muss fachkundig gewesen sein, denn er schreibt neben Personennamen und Satzanfängen auch Rechtsbegriffe groß. In der Literatur hat W. Vogt die Urkunde erstmalig 2000 erwähnt. $^{2}$

\footnotetext{
${ }^{2}$ Wilfried G. VOGT, Bergbau in Breckerfeld, Teil 2; in: Hohenlimburger Heimatblätter 8 , 2000, S. 306-313.
} 
Der Inhalt besagt in der Ich-Form des Evert Stopinch die Übergabe seines gesamten Erbes am Hof Stoupingh uneingeschränkt an Johannes Markt. Eine Begründung fehlt; der Geber bezeichnet sich selbst als gesund. Der Übergabe dürfte die Absicherung eines Darlehens, eine Verschuldung oder Zahlungsunfähigkeit zugrunde liegen. Leider fehlen die Angaben von Zeugen, so dass Zweifel aufkommen könnten, die sich aber ausräumen lassen, weil Johannes Markt oder sein Erbe den Hof der Kirche übereignet hat. Die Urkunde wurde in London ausgestellt, fern der gemeinsamen Heimat.

Gegenstand der Übergabe von 1408 ist der gleichnamige elterliche Hof im Kirchspiel Breckerfeld, ein Einzelhof an der Ennepe nahe der Grenze zum Herzogtum Berg (Stadt Radevormwald), worauf auch der Flurname Schlagbaum in der Hofflur deutet. Der vergleichbare Wegehof in Breckerfeld hatte 1449 einen Wert von 100 Gulden. Zum Vergleich: Die Steuererträge der Stadt (schote) beliefen sich 1449 auf 127 Gulden, 1450 auf 152 Gulden. Der Hof Steupingen wurde nie geteilt und blieb im Eigentum der Kirche, die ihn 1853 für 7300 Taler an den Landwirt J. P. Dörnen verkaufte. ${ }^{3}$ Er diente zuletzt mit einer Pacht von zehn Maltern Hafer, einer Karre Steinkohle und vier Karren Holz jährlich dem Rektorat der kirchlichen Schule. ${ }^{4}$ Das Hypothekenbuch von $1753^{5}$ nennt als Eigentümer die Ev. Lutherische Vicarie zu Breckerfelde, Den Angaben nach auß alter Fundation, wobey die Documenta in denen hieselbst vorgewesenen Feuersbrünsten verbrand seyn, sonsten doch ex antiqua posessione. Offensichtlich war die Urkunde von 1408 in Vergessenheit geraten. Der Wert betrug 400 Reichstaler, d. h. weniger als die Hälfte eines normal großen Bauernhofes von damals 1000 Talern. Im Urkataster von $1825^{\circ}$ wird als Eigentümer das Pastorat (z. T. verändert in Rectorath) genannt. Die Familie Markt war der Kirche sehr verbunden, denn schon 1382 wird ein Johannes up dem Marte als Kirchmeister genannt. Der Name Steuping oder Steupingen bedeutet, dass der Hof zu den ältesten Siedlungen im Raum Breckerfeld gehört, vermutlich schon aus altsächsischer, d. h. vorkarolingischer Zeit. Es handelt sich um einen der südlichsten -ing-Namen in diesem Gebiet zwischen Wupper und Lenne. Der Hof Steupingen wird

\footnotetext{
${ }^{3}$ Anton MEIER, Geschichte und Urkundenbuch des Amtes Breckerfeld: Teil 1 Breckerfeld 1900, Teil 2 Hagen 1908. Nachdruck Hagen 1973, hier Teil 1, S. 75.

${ }^{4}$ Archiv der Evangelischen Kirchengemeinde Breckerfeld, Lagerbuch C3 (alt), 7 (neu) seit 1780.

${ }^{5}$ Staatsarchiv Münster, Gfsch. Mark Gerichte III 5,2. Hypothekenbücher der Grafschaft Mark Nr. 69. Kirchspiel Breckerfeld, Bauerschaften. 1753 (-1806), Hof 92.

${ }^{6}$ Kataster- und Vermessungsamt Ennepe-Ruhr. Schwelm. Flur XXI gen. OberHolthausen, Verzeichnis der Güterbesitzer der Grundgüter und ihres Flächen-Inhalts, Reg.-Bez. Arnsberg, Landräthlicher Kreis Hagen, Gemeinde Breckerfeld (sog. Urkataster 1825/26).
} 


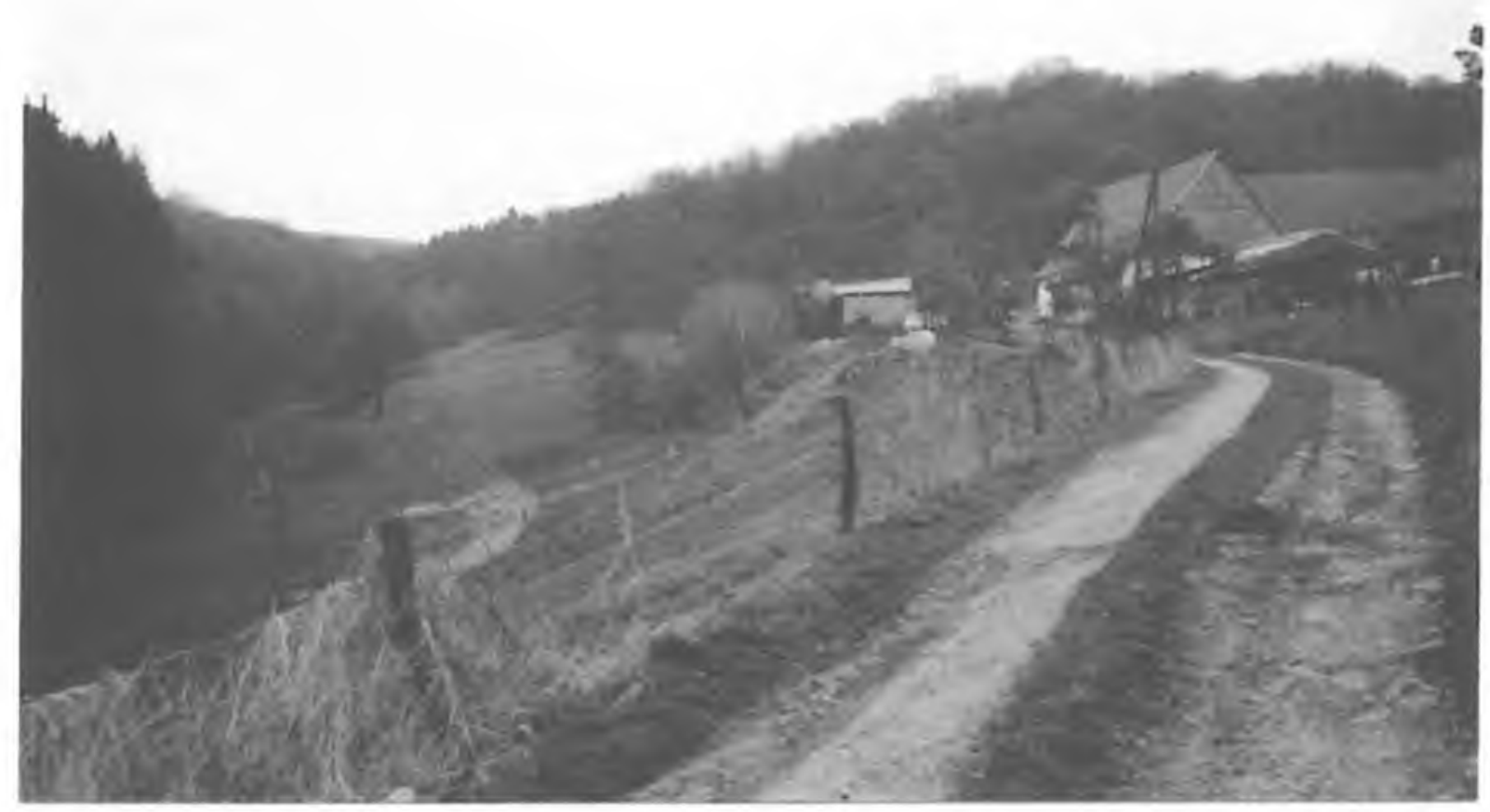

Abb. 1: Der Hof Steupingen 2008.

noch heute landwirtschaftlich genutzt, seit 1916 von der Familie Figge, er umfasste ursprünglich 34 ha.

\section{Die Familien Markt und Stopingh}

Die Prosopographien ,Maerten, up den ', und ,Markte, up dem‘, 229/229.1, geben den Stand des Wissens wieder. ${ }^{7}$ Der Empfänger (Johannes up de Markede, Hans Markett) lässt sich um die Zeit der Urkundenausstellung 1406/07 (zusammen mit Vrowyn Epiynsketh, d. h. Epscheid) und fünfmal 1410 in London nachweisen; zweimal importiert er Stahl ${ }^{8}$ (aus seiner Heimatstadt?). Die Urkunde von 1408 und die Geschichte Breckerfelds liefern weitere Informationen. So wird 1383 ein Johannes up dem Marte als erster unter den sechs Kirchmeistern in Breckerfeld in Zusammenhang mit einem Prozess genannt. ${ }^{9}$ Dabei ging es um die Besetzung der Pfarrstelle, welche die Breckerfelder wie üblich für sich beanspruchten. Der Kölner Erzbischof entschied das Verfahren zu Gunsten der Breckerfelder. 1423

\footnotetext{
${ }^{7}$ Siehe Anm. 1.

${ }^{8}$ Für diese Hinweise - sowie für grundsätzliche Aussagen zur Urkunde U 1 - bin ich Prof. Stuart Jenks/Erlangen, dankbar.

${ }^{9}$ Eduard HELLWEG, Geschichte der evangelischen Gemeinde Breckerfeld, Schwelm 1883; MEIER, Amt Breckerfeld (wie Anm. 3), Teil 1, S. 149
} 


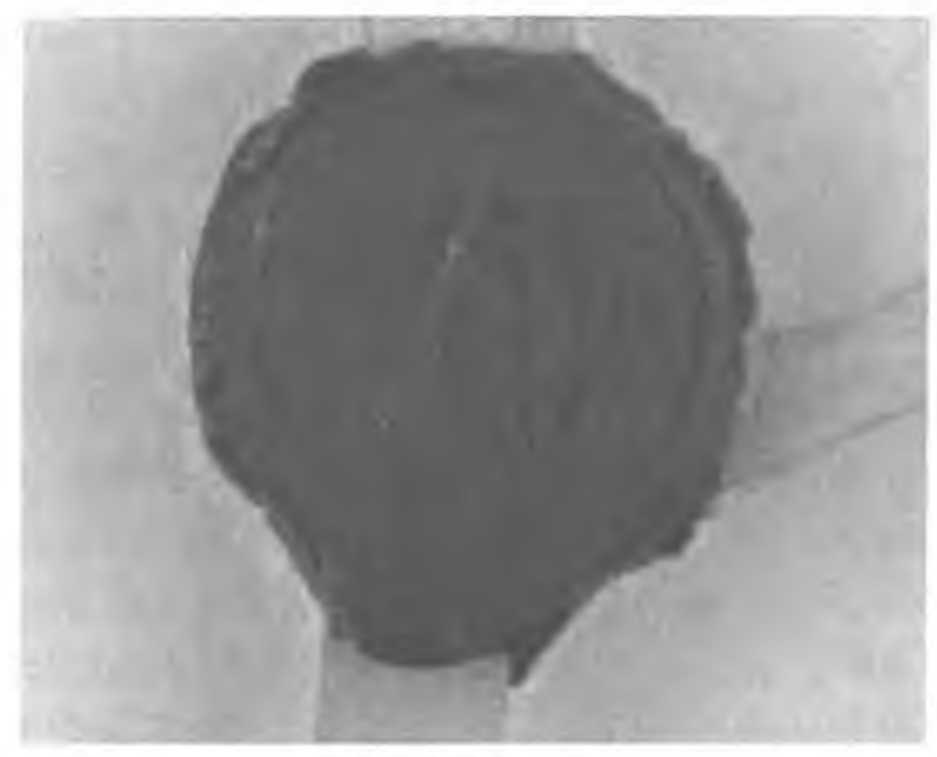

Abb. 2:

Das Siegel des Johan Market von 1423 .

schwor ein Johan Market der Stadt Köln Urfehde, er war Breckerfelder Bürger. ${ }^{10}$ An dieser Urkunde vom 17.7.1423 hängen drei Siegel, von denen das linke von Johan Market ist. Das Siegel zeigt zwei Dolche und ein M. In den Breckerfelder Steuerlisten von 1449/50 taucht die Familie Market nicht auf. Feyerabend nennt etliche up den Marte (o. ä.) für Reval 1368-1443. 1317, 1366, 1384 und 1408 wird die Familie op dem Markete in Iserlohn erwähnt. Demnach liegt hier der Ursprung der Familie, vermutlich schon seit dem 13. Jahrhundert (Iserlohn erhielt vor 1278 Marktrecht.)." 1368 und 1372 vertritt Tideman up dem Markte das livländischgotländische Drittel als Ältermann in Brügge. 1369 ist ein Gert oppe dem Markede in Fellin genannt. Ob die Familie Grafweg ,an dem Marte“ in der benachbarten bergischen Stadt Radevormwald, wo sie z. B. 1556, 1561 und 1567 genannt wird, mit der Iserlohner bzw. Breckerfelder Familie verwandt ist, bleibt sehr fraglich. ${ }^{12}$

Die Prosopographien Scupinc/Stepyng 315/315.1, belegen, dass der Kölner (oder Lübecker) Bürger Frowin Steping (Stepyn, Stopyng, Stupin) 1390 in London Ältermann war; er wird dort 1385-95 genannt, 1379/80 in

\footnotetext{
${ }^{10}$ Meler, Amt Breckerfeld (wie Anm. 3), Teil 1, S. 136. - Historisches Archiv der Stadt Köln. Best. I (HUA) U1/9991. (Sollten die zwei Dolche im Siegel „Abbildungen“ der berühmten „brekervelder" sein?)

"Liselotte FEyERABEND, Die Revaler und Rigaer Familiennamen im 14. und 15. Jh. (Quellen und Darstellungen zur Baltischen Geschichte 7), Köln 1985. - Guido HEINzMANN, Die hansischen Städte im Märkischen Kreis, in: Der Märker 50/, 2001, S. 136 Iserlohn 1366. - Wilhelm SCHULTE, Iserlohn, Die Geschichte einer Stadt, Iserlohn 1937/38, Bd. I, S. 192, Bd. 2, UB S. 7, 17 und 33. (Für diese Hinweise danke ich Dr. Wilhelm Bleicher, Iserlohn.)

${ }^{12}$ Peter Arnold Heuser, Stadt und Pfarrei Radevormwald vom Spätmittelalter bis zum Ende des Dreißigjährigen Krieges (14.-17. Jh.), in: Monatshefte für Evangelische Kirchengeschichte des Rheinlandes 39, 1990, S. 144.
} 
Brügge. In Nowgorod waren Hartwic Stopingh als Ältermann 1374 und Vrowinus (Scupinc, Stepyng) 1375. ${ }^{13}$ Diese Angaben lassen sich durch die Überlieferung in Breckerfeld ergänzen. Dass die Hof- und Familiennamen ursprünglich gleich lauteten, ist für diesen Bereich der Grafschaft Mark typisch. Die Listen der steuerpflichtigen Breckerfelder Bürger von 1449/ 50 zeigen, wie sich in jener Zeit die Wandlung von der Herkunfts- zur Familienbezeichnung vollzog. ${ }^{14}$ Eine Familie Steuping ist für die Stadt nicht belegt. 1483 tritt jedoch ein Hans Stopinge im benachbarten Burg an der Ennepe als Zeuge auf. ${ }^{15}$ Es dürfte sich um den Bewohner bzw. Pächter des gleichnamigen Hofes gehandelt haben. Ob er ein Nachkomme des Evert Stopinch war, lässt sich nicht sagen, vielleicht war der Hofname auch nur auf ihn übertragen. Jedenfalls belegt die Urkunde U 1, dass der in der Prosopographie Nr. 315.1 genannte Testamentsvollstrecker Everard Stepyng, der 1395 den Hof erbte, ihn 1408 übergab. Nach 1483 taucht der Familienname Steuping in Breckerfeld nicht mehr auf. Gegen Ende des 15. Jahrhunderts wird für den Hof Die schulte zo Stoepinck, eine Bergische, als Besitzerin genannt. ${ }^{16}$ Schulte bedeutete seinerzeit Pächter, demnach war der Hof bereits Eigentum der Kirche. Um 1500 saß auf dem Hof kein Bergischer mehr. ${ }^{17}$

\section{Breckerfeld als Hansestadt}

Breckerfeld ist heute eine Titularstadt mit gut 9000 Einwohnern im Südwesten der ehemaligen Grafschaft Mark (Westfalen) südlich von Hagen an der Grenze zum Bergischen (Rheinland). Noch heute liegt der Ort in einer Rodungsinsel, d. h. man kann ihn nur erreichen bzw. verlassen, wenn man durch Wald fährt. Vorgänger der Stadt war das Dorf Altenbreckerfeld (1345 erstmalig so bezeichnet), wie der dortige Tie ausweist; Breckerfeld wird 1184 als vicus erstmalig erwähnt, es gab bereits eine Mühle. ${ }^{18}$ Ein Pfarrer wird $1205,{ }^{19}$ das Kirchspiel $1252^{20}$ zuerst genannt. Die Keimzelle

\footnotetext{
${ }^{13}$ wie Anm. 1. - Birte SCHUbERT, Hansische Kaufleute im Novgoroder Handelskontor: in: Norbert Angermann und Klaus Friedland (Hg.), Novgorod: Markt und Kontor der Hanse, Köln 2002, S. 79-96.

${ }^{14}$ MeIER, Amt Breckerfeld (wie Anm. 3), Teil 1. S. 137/138.

${ }^{15}$ Melter. Amt Breckerfeld (wie Anm. 3), Teil 2. Nr. 29. S. 188.

${ }^{16}$ Meier, Amt Breckerfeld (wie Anm. 3), Teil 1, 1. S. 84

${ }^{17}$ MeIEr, Amt Breckerfeld (wic Anm. 3). Teil 1, S. 85.

${ }^{18}$ Klaus Albert HöLleR, Der südwestlichste Tie Sachsens; in: Hohenlimburger Heimatbläter 11, 2006, S. 361-367; Universitäts- und Landesbibliothek Düsseldorf, Ms. G 5 fol. 87r (sog. Siegburger Mirakelbuch); allgemein: Klaus Albert Höller, Breckerfelder Geschichtsatlas. Ennepetal 2009.

19 Osnabrücker UB, Bd. II, Nr. 25, S. $18 \mathrm{f}$.

${ }^{20}$ Meler, Amt Breckerfeld (wie Anm. 3), Teil 2, Nr. 1, S. $149 \mathrm{f}$.
} 
der späteren Stadt bildete ein märkischer Küchenhof (1449 kokenhove genannt $)^{21}$ mit einer Burg (1413). ${ }^{22}$ Aber auch das Essener Damenstift hatte Anteil mit dem Vaersthof (1220 bzw. 1332), ${ }^{23}$ der Freigraf von Volmarstein mit dem Vedemhove (1449). ${ }^{24}$ (Die Freigrafschaft ging nach 1324 an die Grafen von der Mark über.) Die Kirche soll nach Angaben der Kirchspielsleute $(1383)^{25}$ von ihnen gestiftet und ausgestattet worden sein, erhielt aber auch Dotationen der Grafen von der Mark (Wiedenhof) und von Volmarstein (Großer Zehnt von Hülscheid vor 1470). ${ }^{26}$ Von Stiftungen der Bürger ist die große des Gerwin von Altenbreckerfeld von 1407 bekannt $^{27}$, zu der eine Mühle, ein Hof, zahlreiche Grundstücke und ein Stadthaus gehören; im Testament Gerwins von 1413 werden die Armen der Stadt „und in den Orten der ländlichen Umgebung“ mit 50 rhein. Goldgulden bedacht. ${ }^{28} \mathrm{Im}$ Testament des geborenen Dortmunders und Lübecker Bürgers Gottschalk Wyse von 1362 findet sich auch ein - verhältnismäßig geringes - Legat von $\mathrm{V}$ m. d. lübisch an die Breckerfelder Kirche, ${ }^{29}$ seine Beziehung zu diesem Kirchspiel ist aber nicht weiter bekannt. Die Kirche steht allerdings nach Nennung seiner Verwandten und nach dem Kloster Gevelsberg schon an zweiter Stelle der zahlreichen bedachten Institutionen. Der Urkataster bezeugt 1825 ihren noch ungeschmälerten mittelalterlichen Grundbesitz.

Als Hansestadt wird Breckerfeld gelegentlich erwähnt, so 1469, 1494, 1535,1549 und $1554 ;^{30}$ auf Hansetagen war sie als sog. Beistadt (cleyne bystede) durch die größeren Städte am Hellweg oder durch Köln vertreten. Für ihr Selbstbewusstsein spricht jedoch, dass sie 1469 als einzige der von

${ }^{21}$ Stadtarchiv Breckerfeld. Steuerlisten 1449/50.

22 Niederrheinisches UB. Bd. 4, Nr. 76, S. 84.

${ }^{2.3}$ Beiträge zur Geschichte von Stadt und Stift Essen, 34, 1912, S. 25.

${ }^{24}$ Siehe Anm. 21.

${ }^{25}$ MEIER, Amt Breckerfeld (wie Anm. 3), Teil 1, S. 148ff.

${ }^{26}$ Lagerbuch der Evangelischen Kirchengemeinde Breckerfeld.

${ }^{27}$ MEIER, Amt Breckerfeld (wie Anm. 3), Teil 2. S. 154-159 Nr. 5.

${ }^{28}$ Historisches Archiv der Stadt Köln, Testamente. Testament des Gerwin von Altenbreckerfeld von 1413 .

${ }^{24}$ Archiv der Hansestadt Lübeck, Testament des Godschalcus Wyse von 1362. - Ernst Günther KRÜGER. Die Bevölkerungsverschiebung aus den altdeutschen Stïiten über Lübeck in die Städte des Ostseegebiets, in: ZVLGA 27. 1934, S. 115. - Emil DöSSELER. Die Grafschaft Mark und der deutsche Ostseeraum, in: Der Märker 9 und 10, 1963, S. 225-230 und 304-309 (in Lit. Anm. I nicht genannt). - Im Archiv des Klosters Gevelsberg findet sich kein Hinweis auf die Familie Wyse; vgl. Günter ADERS, Das Archiv des vorm. Zisterzienserinnenklosters und späteren Damenstiftes Gevelsberg. in: Jahrbuch des Vereins für Ortsund Heimatkunde in der Grafschaft Mark 66, 1968, S. 1-179.

${ }^{30}$ MEIER, Amt Breckerfeld (wie Anm. 3), Teil 1, S. 126ff. - Hanserecesse, Abı. II. Bd. 6 , S. 239/Nr. 267. - Luise VON WINTERFELD. Das westfälische Hansequartier, in: Der Raum Westfalen 2.1, hg. von Hermann AuBIN und Franz. PETRI, Münster 1955, S. 255-352; HeINZMANN, Hansische Städte (wie Anm. 11). S. 23-31, 133-139 und 180-188. 


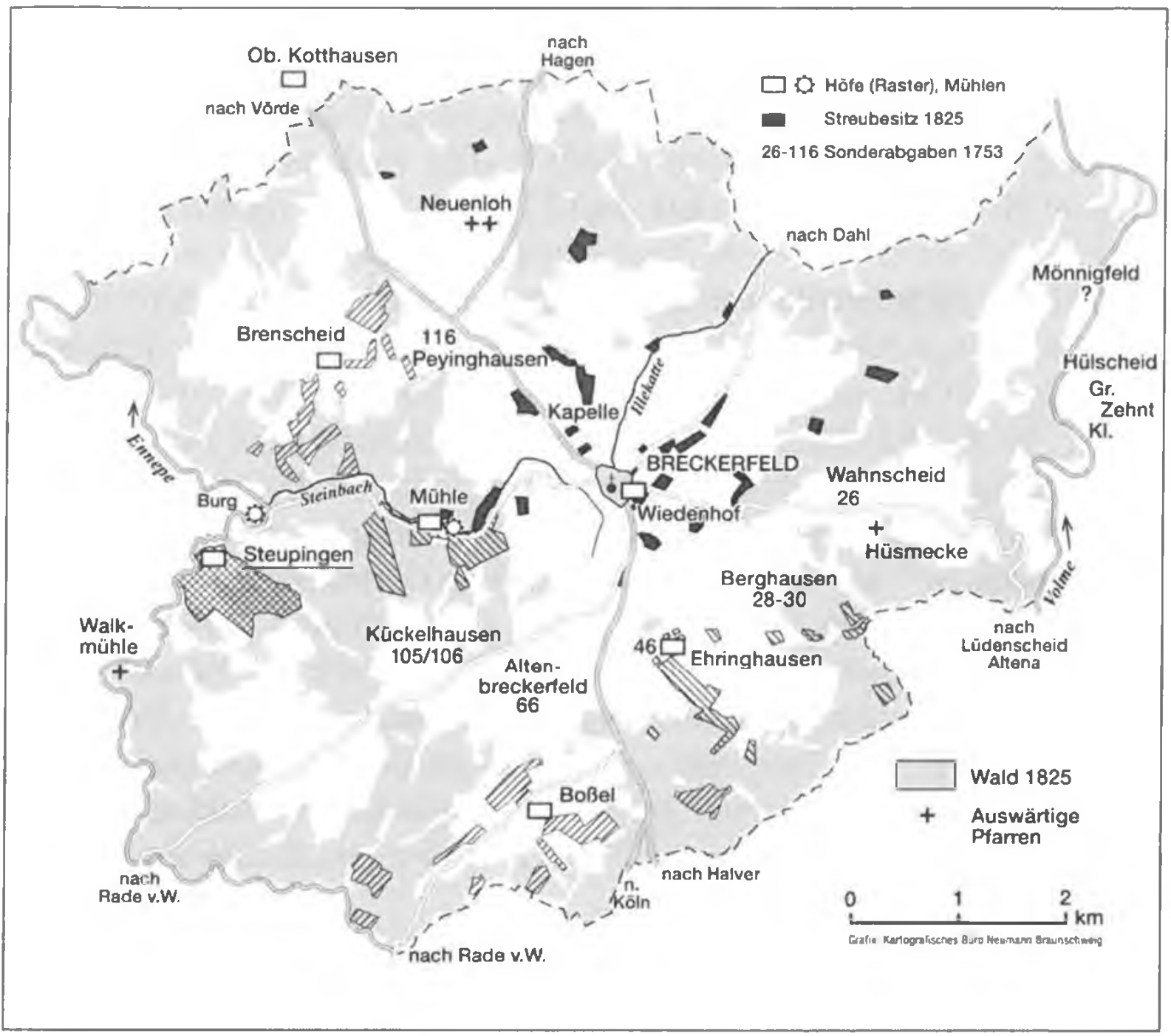

Abb. 3: Das Grundeigentum der Kirche im Urkataster 1825 (Hofflächen schraffiert).

Köln um Unterstützung gebetenen sechs Städte diese verweigerte. 1449/50 hatte die Stadt Breckerfeld ca. 150 steuerpflichtige Bürger. ${ }^{31}$ Sehr auffällig ist das Eigenwahlrecht des Kirchspiels für seinen Pfarrer, welches das besondere Interesse des Erzbischofs von Köln bekundet. In der ganzen großen Erzdiözese Köln gab es neben einigen wenigen Kölner Stadtkirchen sonst kein derartiges Recht für eine Kirchengemeinde. Scheler wertet dies für 1382-85 als ,ein untrügliches Zeichen lebendiger vorstädtischer kommunaler Organisationsformen". ${ }^{32}$ Am Rande von Altenbreckerfeld findet sich ein Tie, eine altsächsische Versammlungs- und Gerichtsstätte, vielleicht die Keimzelle des Selbstbewusstseins der Einwohner, denn weit und breit findet sich kein anderer Tie, außer vielleicht bei Alt-Lüdenscheid (Tyinghausen, Tinghausen).

\footnotetext{
Il Stadtarchiv Breckerfeld, Steuerlisten 1449/50.

${ }^{32}$ Dieter Scheler, Die Stadt Breckerfeld im Spätmittelalter, in: MJfG 104, 2004, S. 28-46, hier S. 30, für die Schmiede-Zunftordnung S. 44-46. - Siehe auch Anm. 18.
} 
Die Breckerfelder hatten als Hersteller von Knüppelstahl und Messern weite Handelsbeziehungen aufgebaut. War zunächst Dortmund das Oberzentrum, in dem spätere Hansekaufleute wie Gerwin von Altenbreckerfeld und Hildebrand Veckinchusen aus (dem benachbarten, erst seit 1308 bergischen) Radevormwald ihre Lehre machten (oder auch heirateten und Bürgerrecht erhielten), so traten an dessen Stelle nach der kostspieligen Dortmunder Fehde 1388/89 das westfälische Soest und vor allem Köln, in der Ferne Lübeck und besonders das Baltikum.

Die Breckerfelder Messer oder Dolche hießen schlicht „brekervelder“ und fanden sogar Aufnahme in das ,Mittelniederdeutsche Handwörterbuch" ${ }^{33}$ Die Zunftordnung für die Stahlschmiede stammt von 1463, für die Messerschmiede von 1545 (Solingen erst 1571, allerdings mit älteren Bruderschaften). Meier hat 1900/08 zahlreiche Quellen dazu veröffentlicht, Kreft diese 2005 in einem größeren Rahmen neu bewertet. ${ }^{34}$ Die Stadterhebung 1396 durch Graf Dietrich von der Mark sollte sicher dem Gewerbe und Handel der Kaufleute dienen, wenngleich im 14. Jahrhundert auch nichtstädtische Kaufleute im Rahmen der Hanse noch Chancen besaßen. Vielleicht handelte es sich um eine direkte Folge des Beschlusses auf dem Hansetag von 1391, das Recht am privilegierten Handel der Hanse auf Bürger von Hansestädten zu beschränken. Das Stadtrecht kam jedoch einerseits als Maßnahme gegen die Abwanderung um Jahrzehnte zu spät, andererseits konnte es den Vorteil der großen Hansestädte nicht ausgleichen. Für die Hansekaufleute Breckerfelds wollte sich auch Graf Gerhard einsetzen, welcher der Stadt besonders wohl gesonnen war und nahebei in Epscheid sein "Junckers guth" hatte. 1449/50 gestattete er der Stadt, den Bergfried seiner Burg für den Bau der Stadtmauer abzubrechen..$^{35}$ 1438-40 machte Graf Gerhard eine Reise nach Livland ${ }^{36}$ und erhielt dafür vom Hochmeister des Deutschen Ordens in der Marienburg einen Geleitbrief für den Fürsten von Nowgorod zu einer Reise ins Heilige Land (!). Sie endete aber unglücklich in Neuschloß am Peipus-See mit der Ermordung seines Dolmetschers Herman tor Koken (1514 Platzname „tor koken“ bei der Burg in der Stadt

\footnotetext{
3, August LüBBEN und Christoph WALTHER, Mittelniederdeutsches Handwörterbuch, Leipzig 1888, Nachdruck Darmstadt 1989, hier S. 66. Zur möglichen Gestalt dieses Dolches s. Abb. 2.

${ }^{34}$ MEIER, Amt Breckerfeld (wie Anm. 3), Teil 2, Nr. 20, S. 176-179, und Nr. 24-40, S. 183-200; Thomas KREFT, Das mittelalterliche Eisengewerbe im Herzogtum Berg und in der südlichen Grafschaft Mark, Diss. Aachen 2001. Herzogenrath 2002, hier S. 302-328, S. 370 für Werden; Scheler, Stadt Breckerfeld (wie Anm. 32), S. 38 f. und 44.

${ }^{35}$ Stadtarchiv Breckerfeld, Steuerlisten 1449/50.

${ }^{36}$ Liv-, Est- und Kurländisches UB (LEKUB), Bd. 9, für die Jahre 1436-43 zahlreiche Nennungen; Nachdruck Aalen 1981.
} 


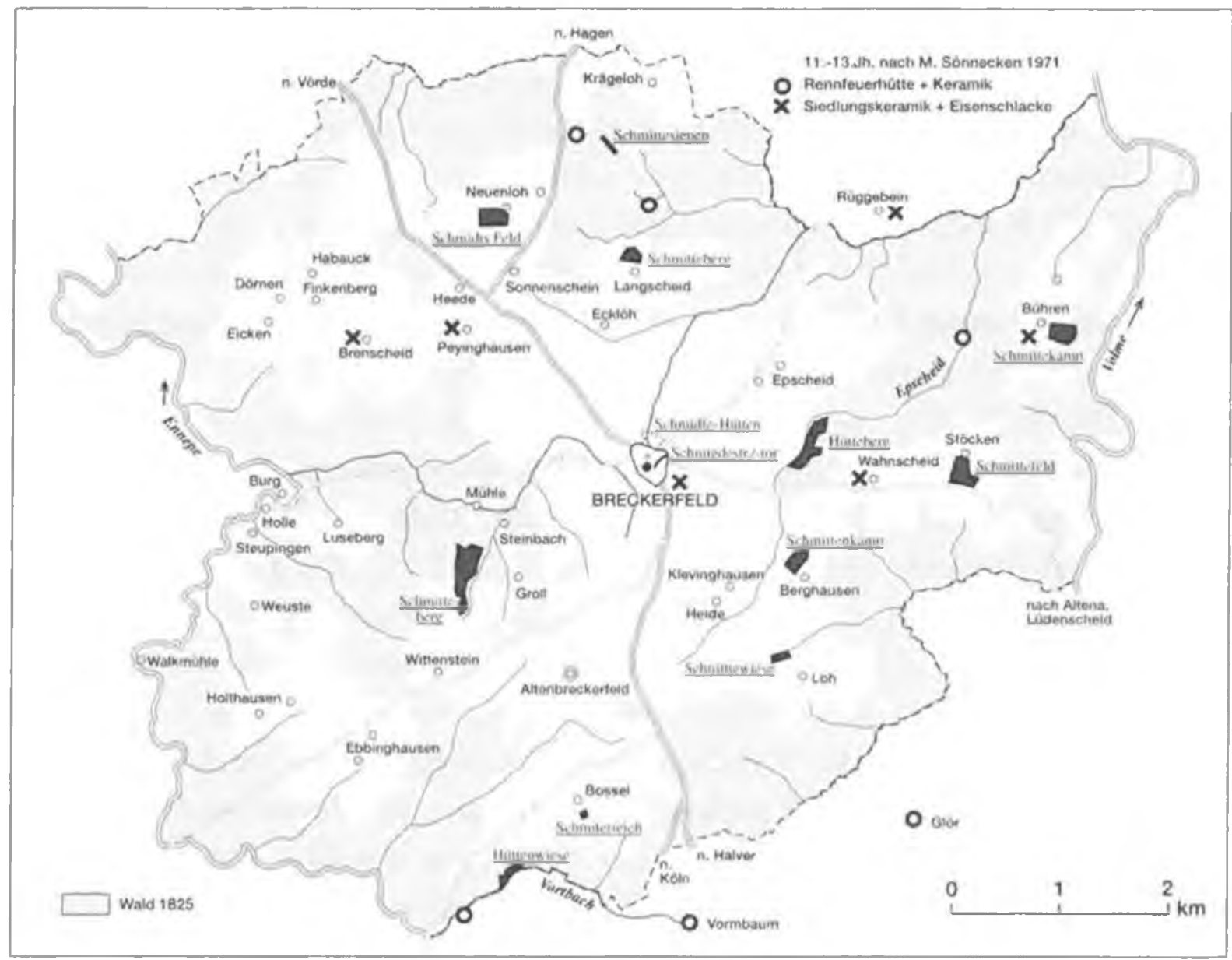

Abb. 4: Rennöfen, Schmiede- und Hütten-Flurnamen (schwarze Flächen) in und bei Breckerfeld.

Breckerfeld). Die Hansestädte und der Landmeister setzten sich nicht entschlossen für den Grafen ein, der Entschädigung von den Russen forderte. Das Einvernehmen mit dem mächtigen Nachbarn scheint ihnen wichtiger gewesen zu sein als ein kleiner Graf aus Westfalen (der 1461 ohne Nachkommen starb).

Materielle Grundlage der Eisengewinnung und -verarbeitung bildeten zunächst das an vielen Stellen vorkommende Eisenerz (später wurde auch Roheisen angekauft) sowie etwa seit der zweiten Hälfte des 13. Jahrhundert die Wasserkraft der zahlreichen Bäche für den Antrieb von Blasebälgen. Etliche Flurnamen zeugen noch heute von der Verbreitung des Schmiedegewerbes in den Bauerschaften. Damit ist die Entstehung der Messeranfertigung noch nicht erklärt. Könnte es sich (wie bei Solingen vermutet) um Kölner Einfluss gchandelt haben? Auch das im Kirchspiel Breckerfeld mehrfach begüterte Kloster Werden kommt als Anreger infrage, denn seit 1414/17 sind dort Mesmeker bekannt. Die aus Iserlohn stammende Familie up dem Marte mit den beiden Dolchen im Siegel deutet damit ebenfalls auf einen möglichen Ursprung, zumal schon 1431 in Iserlohn ein Mesmeker erwähnt wird. 
Wie die Einwohner-Steuerlisten von 1449/50 zeigen, stammte damals etwa die Hälfte der Stadtbürger von den umliegenden Höfen. ${ }^{37}$ Die Karte verdeutlicht das enge nachbarschaftliche Verhältnis durch die Ballung der Hansennamen, deren Verbreitung in der Fläche und deutlich über das Kirchspiel hinaus. Ob die Gewerbetreibenden und die Hansekaufleute zunächst ausschließlich und nach 1396 immer noch zum großen Teil nachgeborene Bauernsöhne waren, ist anzunehmen. Einige waren bis 1315 Freileute auf Freigütern der Freigrafen von Volmarstein (danach bergische bzw. märkische), wie Epscheid und Feckinghausen, andere Höfe gehörten Klöstern oder Stiften, wie Lausberg/Luseberg dem Kloster Werden, andere dem Adel, wie in Boßel/Borstal den Grafen von Volmarstein. Für etwa 40 Höfe lässt sich jedoch keine Grundherrschaft nachweisen, so dass eine frühe Eigenbehörigkeit anzunehmen ist, wie besonders für Altenbreckerfeld und z. B. auch für Steupingen. Evert Stopinch konnte deshalb seinen Hof frei und eigenständig vermachen. Mit diesem frei verfügbaren Eigentum besaßen die Hansekaufleute der Familie Steuping Eigenmittel, um den Start und den Verlauf ihrer Geschäfte selber zu finanzieren bzw. zu sichern - allerdings konnten sie auch alles verlieren!

$\mathrm{Zu}$ den aus dem Umland stammenden Einheimischen traten im 14./15. Jahrhundert Auswärtige, deren Familiennamen auch sonst im weiten Hansegebiet vorkamen. So nennt Meier als Pächter (erbliches Mannlehen) der Grafen von Volmarstein die Krowel und Vuncke (auf Boßel) sowie Reyse (auf Langscheid), Helmich war Vikar, Hanneboill (aus Attendorn ?) Bürgermeister 1426 und Fluiß (vom Niederrhein) in der Stadt, nicht zuletzt die Familie up dem Markete aus Iserlohn. 1449/50 finden sich unter den Stadtbürgern die Löper-Familie Hunt (dreimal), die Bertold. Smalt (als Löper), Sasse, Peppersack (als Vikar), Lympermann (zweimal), Kuckuck, Kruckener, Hardensteyn und Stenverter. Es gingen also nicht nur Breckerfelder als Kaufleute in den weiten Raum der Hanse, es siedelten sich hier auch Auswärtige an.

Da die Hofnamen in Ortsverzeichnissen nur mühsam oder gar nicht zu finden sind, blieb bisher ihre Lokalisierung ein schwer zu lösendes Problem. Zudem weicht die mittelniederdeutsche Schreibweise in etlichen Fällen erheblich von der heute amtlichen ab. Zwar sind manche Hofnamen wie Brenscheid, Bühren. Dahlhausen und Loh weit verbreitet, aber u. a. Epscheid, Wahnscheid. Peyinghausen und Klevinghausen (in Breckerfeld), Halverscheid und Othmaringhausen (Halver), Remlingrade und Feckinghausen (Radevormwald), Störring und Jellinghausen (Vörde), Rüggebein

\footnotetext{
${ }^{37}$ Stadtarchiv Breckerfeld. Steuerlisten 1449/50; MEIER. Amt Breckerfeld (wie Anm. 3), Teil 1, S. $137 \mathrm{f}$.
} 
$+\quad$ urkundlich gesichert

$=$ Stadtbürger 1449/50

* $\quad$ solitäre Namen

- Grenze bis 1929

Grenze nach 1970

() Siedlungs- = Hansenname

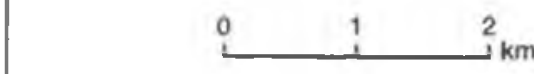
$2 \mathrm{~km}$

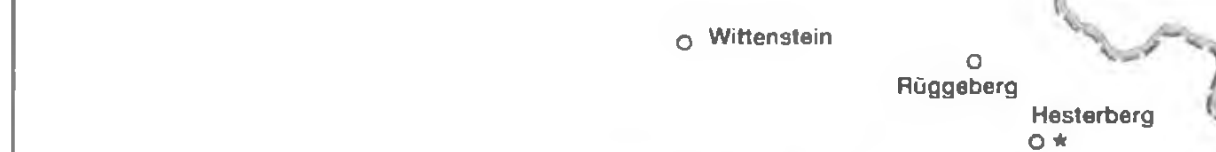

Jellinghausen ${ }^{\star}$

Stóring

t vőrde $=$

ivom Ort =

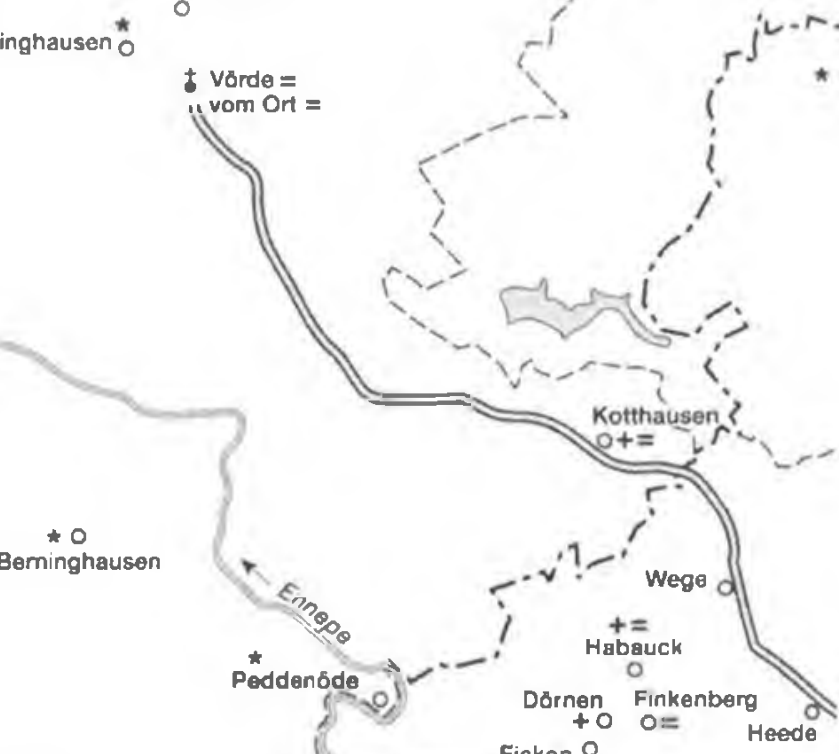

Eicken 0

Brenscheid

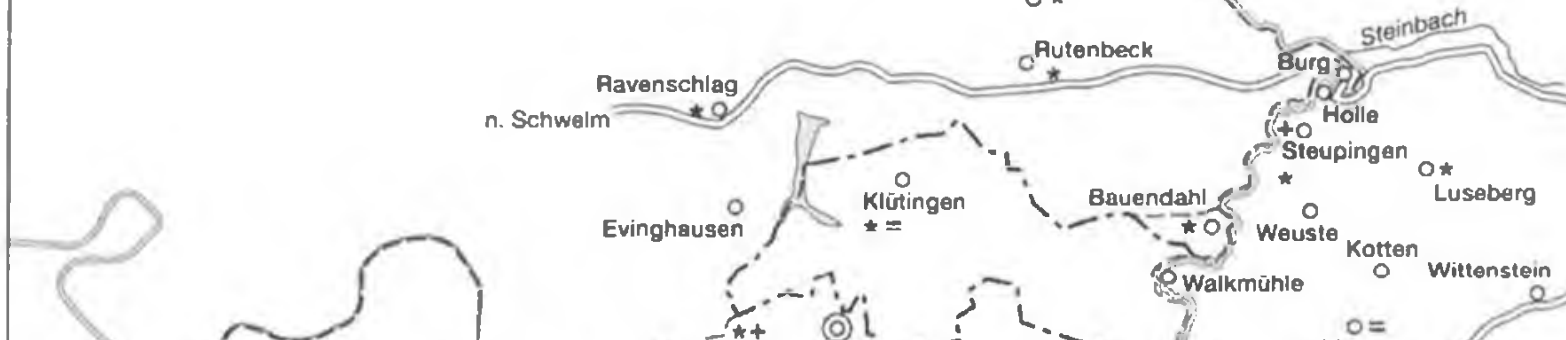

s.
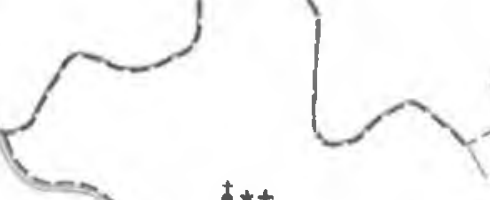

5 Feckinghausen'- - L'

(i)

$0=$
Holthausen

o Obernhof

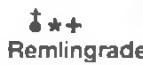

$\stackrel{0}{\circ} \quad \stackrel{0 *}{\text { Wönkhausen }}$

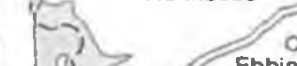

0
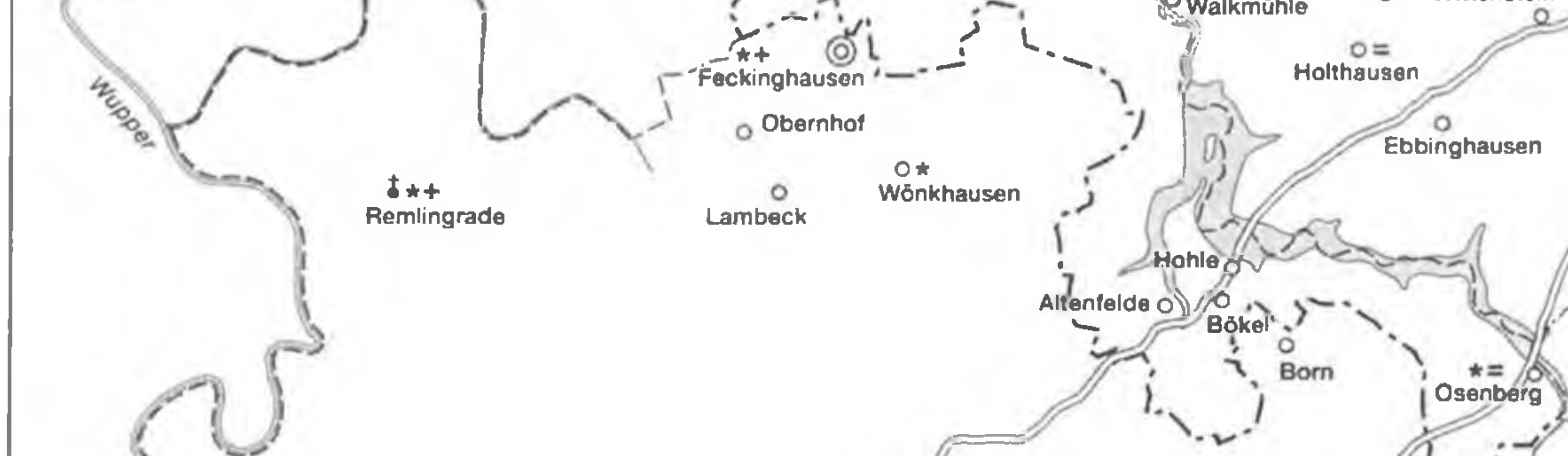

Lambeck
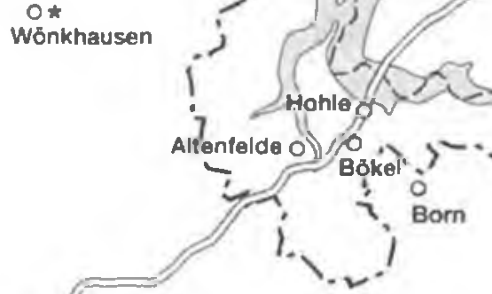

Ebbinghausen

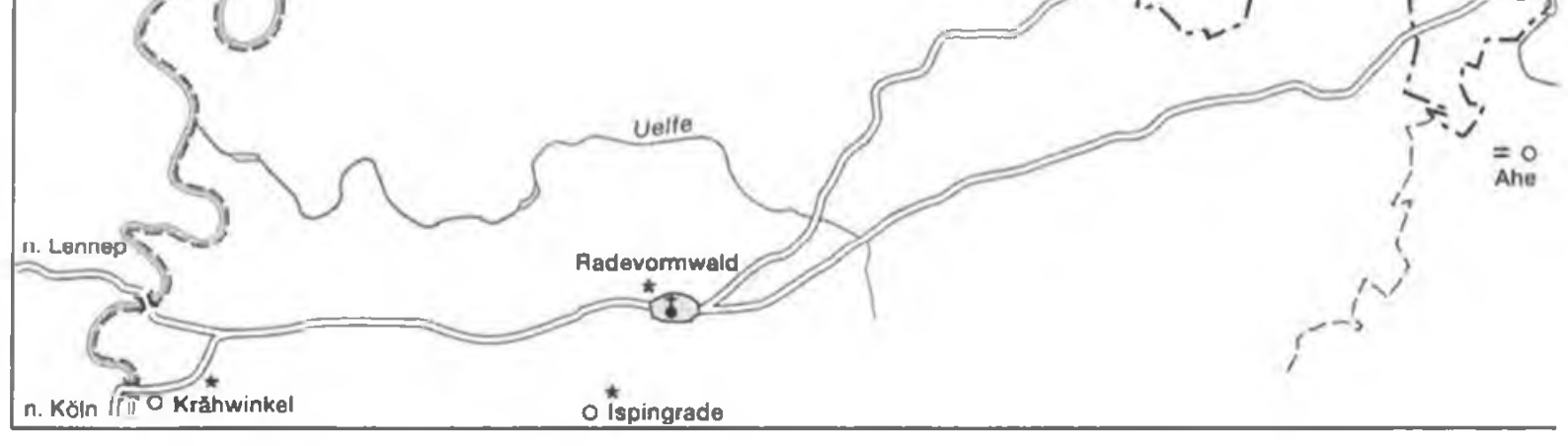

Abb. 5: Wohnplätze, deren Namen mit den Familiennamen von Hansekaufleuten identisch sind. 


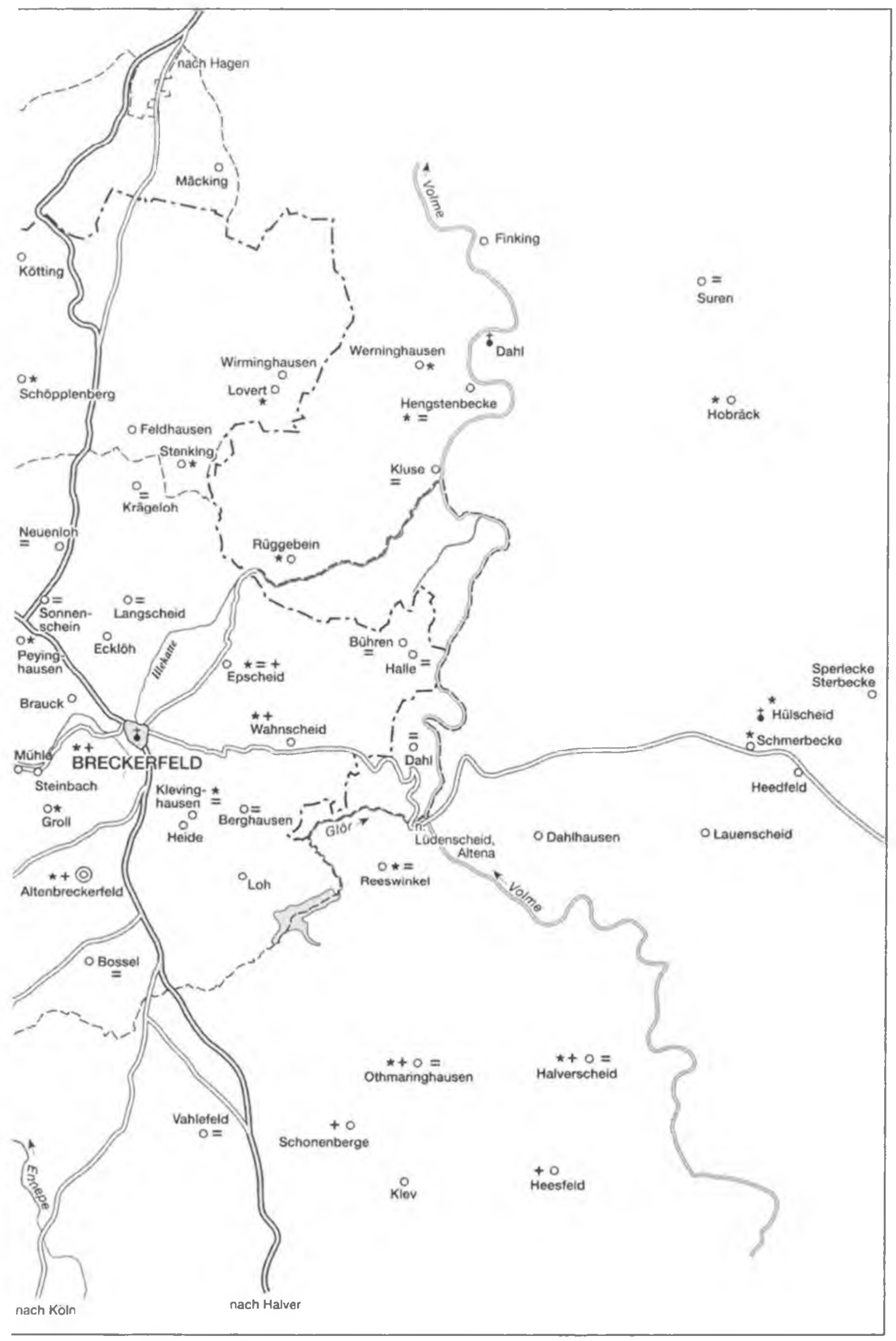


und Werninghausen (Dahl) z. B. gibt es nur einmal und nur in Breckerfeld und Umgebung (,singuläre Namen“ vgl. Karte). Im Lichte dieser Verbreitung und Verortung muss das Bild des Hansekaufmannes auch gesehen werden. Wenn 1386 der Überläufer C. Kelmer (Dortmund/London) sagte, „man könne alle deutschen Kaufleute unbesorgt totschlagen, da sie alle von Dörfern kämen“, dann dürfte er auch Leute wie von den Breckerfelder Höfen gemeint haben. ${ }^{38}$

Die Erträge der Höfe, welche zwar an sich ursprünglich groß genug waren, blieben wegen der kalten, nassen und steinigen Böden gering, und die nicht erbenden Söhne mussten sich anderweitig nach einem Erwerb umsehen, vor allem im Schmiedehandwerk. Erstaunlich bleibt aber dennoch, dass aus einer auch heute noch abgelegenen Gegend so viele junge Männer sich in die Ferne trauten. So sind z. B. in Dorpat - neben Reval eine der Hochburgen von Hansekaufleuten aus Breckerfeld und Umgebung - für 1338-60 T. Rutenbeke, für 1363-97 die van der Borch (Bürgermeister = BM), Eppeschede, Ebbinchusen, Rutenbek, Ekelö, Bredenschede, Rocesberch, Woynchusen, Oldenbrekelfelde und Holthusen bezeugt, für 1406-59 die Eppenschede (BM), Dalhusen, Oldenbrekelvelde $(\mathrm{BM})$, van me Koten, vam Hole (BM), Bredenschede, Luzeberch (BM), Wantschede, Cothusen, Luzeberch (BM), von den Eken, Woeste, van dem Hole, Bredenschede, Cluseman, Eppinchusen, Sasse und Wantschede (BM), für 1456-1510 die Sasse, Wantschede (BM), Borstel, Clevinchuis, Wantschede, Woeste, Borstelen, Eppenschede (BM), Hunt, Clevinchuszen, Holthusen, Limpermann und vam Lo. ${ }^{3 ! \prime}$ Nicht nur für Dorpat, sondern allgemein gilt jedoch, dass nur wenige Familien länger als eine oder zwei Generationen im Ausland im Hansehandel nachzuweisen sind.

Die Bewohner der angrenzenden Kirchspiele gingen nach Breckerfeld, auch um in dieser Stadt das Bürgerecht zu erhalten: so z. B. aus Halver die Halverscheid und Hersfeld, aus Radevormwald die Osenberg und Klüting, aus Vörde die vom Orde und Korthaus, aus Waldbauer die Kötting, aus Dahl die Klusemann und Hengstenbecke sowie von jenseits der Volme die Suren und Wesselberch.

Eine Reihe dieser Hansekaufleute sind durch ihre Testamente oder den Schriftwechsel als Bürger aus Breckerfeld oder Umgebung gesichert. So

${ }^{38}$ Klaus FRIEDLAND, Hansische Handelspolitik und hansisches Wirtschaftssystem im 14. und 15. Jahrhundert; in: DERS. (Hg.), Frühformen englisch-deutscher Handelspartnerschaft (Quellen und Darstellungen zur hansischen Geschichte) 1976, S. 87-106, hier S. 92.

${ }^{39}$ Siehe im Register des LEKUB (wie Anm. 36) unter den entsprechenden Namen; Literatur s. Anm. 11; außerdem Fritz RörIG, Hansische Beiträge zur deutschen Wirtschaftsgeschichte. Breslau 1928, hier S. 230 \%u T. Rutenbeke; Robert Arthur voN LEMM, Dorpater Ratslinie 1319-1889 und das Dorpater Stadtamt 1878-1918 (Wissenschaftliche Beiträge zur Geschichte und Landeskunde Ost-Mitteleuropas 48), Marburg 1960. 


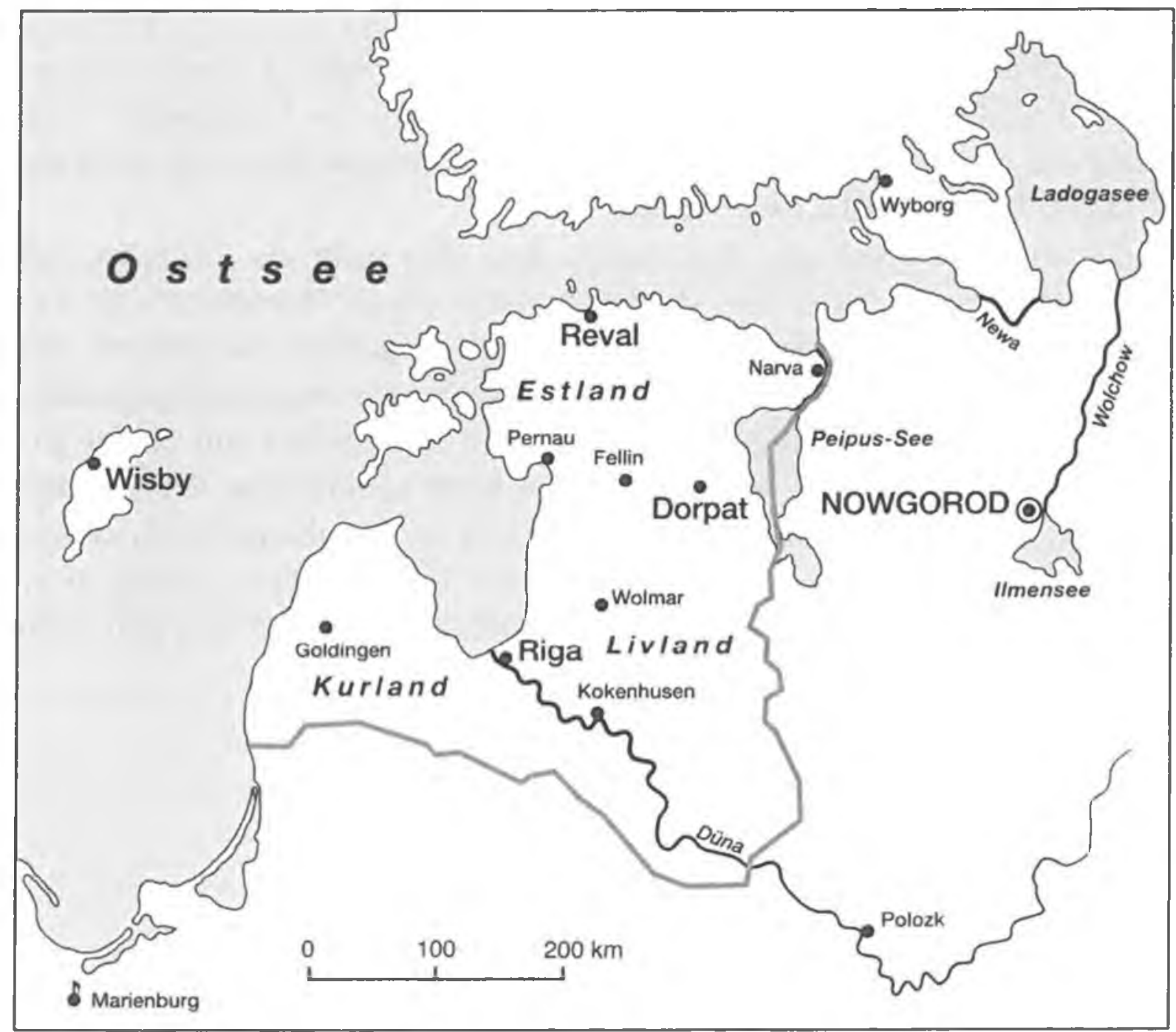

Abb. 6: Aufenthaltsorte von Hansekaufleuten aus Breckerfeld und Umgebung im Ostseeraum.

schrieb Breckerfeld am 17.1.1426 an Reval wegen des Testamentes der Familie Otmerinchusen und am 25.7.1433 wegen der Familie Halverschet. Auch hatte der Breckerfelder Bürgermeister Hanneboill einen Verwandten im Revaler Rat. Andere Belege - wie Woeste mit Feckinghausen oder Jellinghaus mit vom Orde - ergeben sich durch Verwandtschaft. In vielen Fällen dürften aber nur die Vorfahren aus Breckerfeld stammen und sie selber schon außerhalb geboren sein, so der mit Hildebrand Feckinghausen (Veckinchusen) eng zusammen arbeitende Thidemann Breckefeld (wohl aus Lüneburg) und 1475/76 der aus Soest stammende Londoner Ältermann Arnold Breckervelde sowie 1452 und 1469 der Soester Bürgermeister Ewald Breckelveld. ${ }^{41}$ Selbst in diesen Fällen werden aber die freundschaftlichen Beziehungen und kaufmännischen Geschäfte durch eine gemeinsame Herkunft und Abstammung gefördert worden sein. Fahlbusch

40 KREFT. Einsengewerbe (wie Anm. 34), hier S. 318-321. 
beschreibt für die Zeit um 1470, worum es wohl auch den Breckerfelder Hansekaufleuten ging: territoriale Zugehörigkeit, Platz in einem interurbanen Familienverband und die existentielle Basis im Fernhandel. ${ }^{41}$ Als Beleg für eine Zusammenarbeit in der fernen Fremde lässt sich auch die Urkunde U1 vom 3.11.1408 werten.

Zusammenfassend kann man sagen, dass über mehr als 150 Jahre von der Mitte des 14. bis in das 16. Jahrhundert hinein Hansekaufleute aus Breckerfeld und Umgebung in großer Zahl im ganzen Hanseraum von England bis Nowgorod tätig waren und dabei auch verantwortungsvolle $\ddot{A}$ mter bekleideten. Darüber hinaus gehörte Breckerfeld mit seiner Erzeugung von Stahl und Messern zu den binnenländischen Wirtschaftszentren, die vor allem über Köln und Lübeck am Fernhandel teilnahmen. Die Bedeutung Breckerfelds als Hansestadt blieb dagegen gering, trotz eines regen Zuzuges aus den Nachbargemeinden und auch aus dem weiten Hansegebiet.

${ }^{41}$ Friedrich Bernward FAHLBUSCH, Regionale Identität. Eine Beschreibungskategorie für den hansischen Teilraum Westfalen um 1470?; in: HGbll. 122, 1994. S. 139-159. 